\title{
Charge characteristics of the MOS structures with oxide films containing Si nanocrystals
}

\author{
E.V. Begun, O.L. Bratus', A.A. Evtukh, E.B. Kaganovich, E.G. Manoilov \\ V. Lashkaryov Institute of Semiconductor Physics, NAS of Ukraine \\ 41, prospect Nauky,03028 Kyiv, Ukraine, e-mail:dept_5@isp.kiev.ua
}

\begin{abstract}
The processes of charge accumulation in the MOS structures with $\mathrm{SiO}_{2}$ films containing $\mathrm{Si}$ nanocrystals are investigated, depending on the conditions of their formation by pulsed laser deposition. High-frequency capacity-voltage characteristics of structures with the different thicknesses of films, sizes of Si nanocrystals, and their densities in the case of doping the films with gold and without it are measured. It is shown that the positive and negative charges are built-in, respectively, in the undoped films and those doped with gold. At the record of $C-V$ curves, the accumulation of a positive charge is observed. The value of accumulated charge is higher in thin films and in the films doped with gold. The obtained results testify the possibility of the use of pulsed laser deposition for creation of memory structures based on the charge capture by Si nanocrystals.
\end{abstract}

Keywords: silicon, quantum dot, capacity-voltage characteristics, non-volatile memory.

Manuscript received 30.03.07; accepted for publication 24.04.07; published online 19.10.07.

\section{Introduction}

From the beginning of the 1990s, the intensive researches and developments of structures on the base of the nanocomposite system, silicon nanocrystal - silicon dioxide ( $\mathrm{Si} \mathrm{NC} / \mathrm{SiO}_{2}$ ), have been performed with the purpose to create light emitting sources in the visible region of spectrum, photodetectors, and memory devices on silicon (c-Si). This nanocomposite is silicon with the electronic system of dimension 0D (quantum dots) and is often named nanocrystalline silicon (nc-Si). Important information about the processes of capture of charge carriers, the current flow, the bands diagram of the $\mathrm{M} / \mathrm{nc}-\mathrm{Si} / \mathrm{c}-\mathrm{Si} / \mathrm{M}$ structures is given by the studies of their electric properties, in particular, capacity-voltage $(C-V)$ and current-voltage $(I-V)$ characteristics. The results of studies of the capacity properties of structures with nanosize silicon are generalized in a review [1]. As a rule, $C-V$ characteristics with the shape similar to that for the MOS structures are observed.

Interest to the charge characteristics of these structures is related foremost to developments of devices of memory, working on the capture of charge carriers by sites (traps, NC). They possess the prospect of reduction of the tunnel oxide thickness. At the creation of the memory structures based on a floating gate with $\mathrm{Si} \mathrm{NC}$, there are the tasks of control over Si NC sizes, their dispersion, density, and the density of electronic states at the interface of c-Si substrate and dielectric. Most works concern the structures containing porous silicon (por-Si) or layers of $\mathrm{SiO}_{2}$ with implanted silicon.

The method of pulsed laser deposition (PLD) of films is characterized by the large technological flexibility, advantages of vacuum cleanness, controllability of the erosive torch parameters, congruence of the process, compatibility with silicon technology, etc. It allows one to obtain $\mathrm{SiO}_{2}$ films with quantum-size $\mathrm{Si} \mathrm{NC}$ characterized by effective photoluminescence (PL) in the visible region of the spectrum at room temperature [2]. But, to our knowledge, the charge characteristics of the MOS structures based on these films are not practically studied. Purpose of the present work is to investigate the charge characteristics of $\mathrm{M} / \mathrm{nc}-\mathrm{Si} / p-\mathrm{Si} / \mathrm{M}$ structures with the nc-Si films obtained by PLD in dependence on conditions of their formation.

\section{Experiment}

The Al/nc-Si/p-Si structures containing nc-Si films on $p$ Si substrates $(\rho=10 \mathrm{Ohm} \times \mathrm{cm},(001))$ were formed at the nc-Si film deposition by PLD in a vacuum chamber with residual gas pressure $10^{-3} \mathrm{~Pa}$ for the size separation of $\mathrm{Si}$ particles and the formation of films with a mirror surface [3].

A target was single crystalline silicon with a preliminarily deposited gold film of $100 \mathrm{~nm}$ in thickness 
on its surface or without it. Doping by gold atoms was carried out for passivation of the unsaturated dangling bonds of silicon in the film and the formation of $\mathrm{SiO}_{2}$ instead of $\mathrm{SiO}_{x}(x<2)$. Introduction of gold resulted in the increase of the middle sizes $\mathrm{Si} \mathrm{NC}$ and their dispersion $[3,4]$. The YAG: $\mathrm{Nd}^{3+}$ laser beam (wavelength $1.06 \mu \mathrm{m}$, energy and duration of pulses $0.2 \mathrm{~J}$ and $8 \mathrm{~ns}$, respectively, and frequency of their repetition $25 \mathrm{~Hz}$ ), working in the Q-switched mode, scanned the target. The film deposition velocity lay in the range 2$20 \mathrm{~nm} / \mathrm{min}$, and the thicknesses of films were in the range $50-500 \mathrm{~nm}$. With removing from the erosive torch axis, the film thickness decreased, as shown in Fig. 1. The films grown at large distances from the torch axis were characterized by the less sizes of Si NC and their higher concentration (see the insertion in Fig. 1). These regularities of formation of the $\mathrm{SiO}_{x}$ films with $\mathrm{Si} \mathrm{NC}$ structure are a result of their deposition from the back low-energy flow of erosive torch particles [3]. The sputtering of aluminium films on the frontal and rearward surfaces completed the formation of MOS structures. The laser annealing provided the ohmic Al contact to $p$-Si substrate.

The time-resolved PL spectra were measured in the energy range 1.4-3.2 $\mathrm{eV}$ at excitation by the radiation of a nitric laser (wavelength $337 \mathrm{~nm}$, and pulse duration $8 \mathrm{~ns}$ ) with stroboscopic registration of a signal in the photon counting mode. The strobe width was $250 \mathrm{~nm}$. The spectra of photoluminescence, successive in time, were measured with delay of the strobe in relation to the pulse of a laser up to maximal times of PL relaxation about $20 \mu \mathrm{s}$.

Measurements of $I-V$ characteristics were performed in darkness at applying the voltage changing on increasing and falling behaviors with a step of $0.1 \mathrm{~V}$. The range of measured currents was $5 \times 10^{-11} \ldots 10^{-2} \mathrm{~A}$.

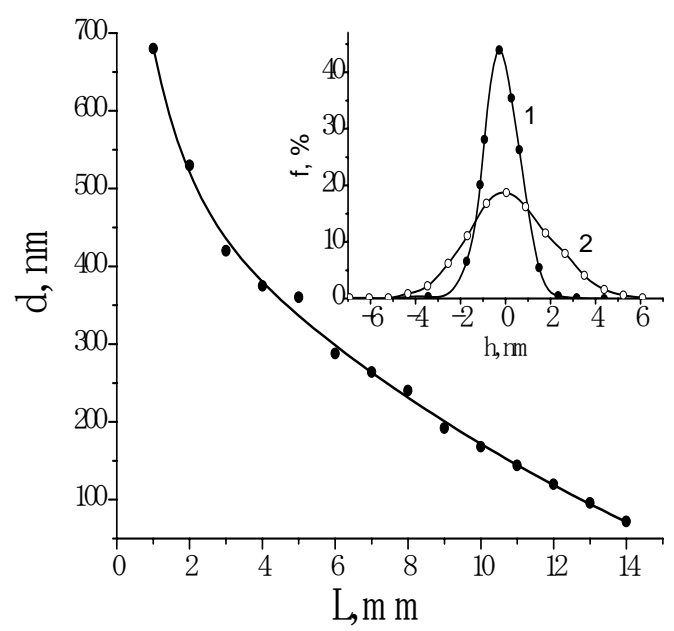

Fig. 1. Dependence of nc-Si film thickness on the distance from an erosion torch. In insert: histograms of the height distribution of surface asperities at a distance of $12 \mathrm{~mm}$ from the torch (1) and nearby the torch (2). Results of Atomic Force Microscopy [4].
The $C-V$ characteristic measurements were fulfilled with an AMTs-1530 C meter at a testing signal frequency of $1 \mathrm{MHz}$ and a voltage rate of $50 \mathrm{mV} / \mathrm{s}$ in the interval from -15 to $+15 \mathrm{~V}$. The measurement of $C-V$ characteristics begins from the voltage corresponding to the depletion or inversion of the surface semiconductor layer (in this case of $p$-type semiconductor - from positive gate voltages) to voltages corresponding to the accumulation, and in the reverse direction. The highfrequency signal amplitude was $20 \mathrm{mV}$.

\section{Results and discussion}

The PL spectra of obtained films lay in the interval of radiation energies of 1.4-3.2 eV, and the times of PL relaxation lay from tens of nanoseconds to tens of microseconds. Undoped films show the PL spectra shifted to the high-energy spectral region and are characterrized by rapid, less than $250 \mathrm{~ns}$, PL relaxation times. The PL spectra of the films doped with gold are located in the low-energy spectral region, and the slow microsecond PL relaxation is inherent to them (Fig. 2). The resulted PL spectra of obtained nc-Si films are typical of the nanocomposite structures $\mathrm{Si} \mathrm{NC} / \mathrm{SiO}_{2}$. Their photoluminescence properties, as was shown earlier in [3], are caused by radiative annihilation of excitons in quantum-size $\mathrm{Si} \mathrm{NC}$ with the barrier $\mathrm{SiO}_{2}$ layer.

$I-V$ characteristics of the investigated structures $\mathrm{Al} / \mathrm{nc}-\mathrm{Si} / p-\mathrm{Si} / \mathrm{Al}$ had a rectifying character: the forward direction of the current corresponds to a positive potential on $p$-Si (Fig. 3). The forward branch of the $I-V$ characteristics at a bias from the parts of one volt to a few volts with excluding the voltage drop on a ballast resistance corresponds to the exponential dependence. At a bias of more than $5-10 \mathrm{~V}$, the saturation in the current was observed, and the current density achieved

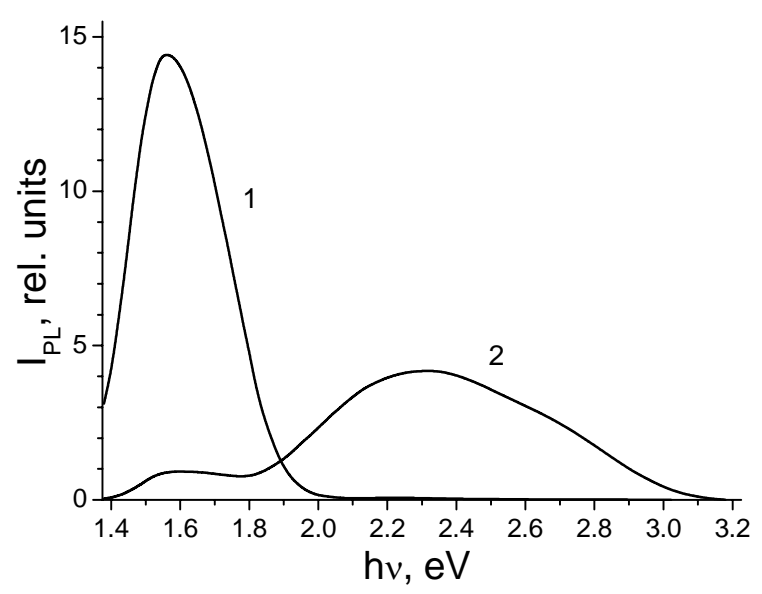

Fig. 2. Time-resolved PL spectra of the films doped with gold (1) and undoped (2). 


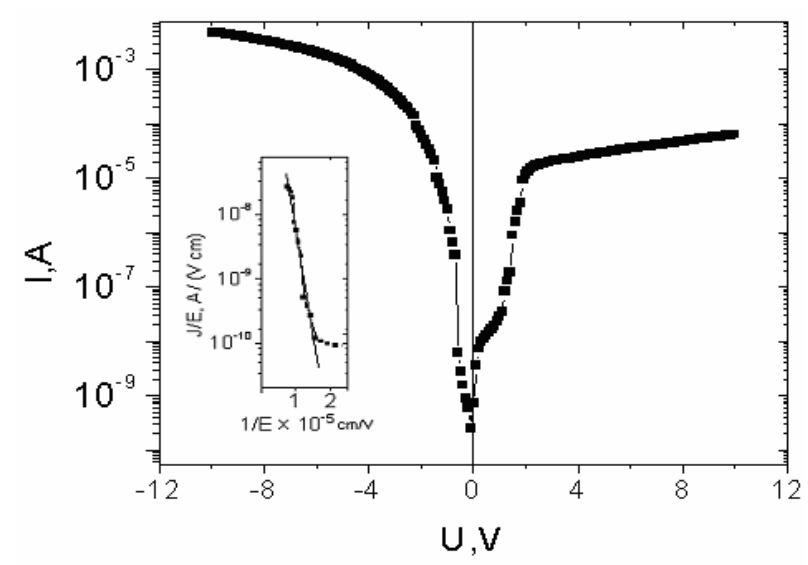

Fig. 3. Typical $I-V$ characteristics of the MIS structure with nanocomposite $\mathrm{Si} \mathrm{NC}-\mathrm{SiO}_{2}$ film $(d=173 \mathrm{~nm})$. In the insert: direct branch in the Abeles $(\ln (I / E)-1 / E)$ coordinates.

the values $10^{-1} \ldots 10^{-2} \mathrm{~A} / \mathrm{cm}^{2}$. At electric fields $E=(0.5 \ldots 1) \times 10^{5} \mathrm{~V} / \mathrm{cm}$, the significant parts of $I-V$ characteristics correspond to the direct tunnelling mechanism between QD through the $\mathrm{SiO}_{2}$ potential barriers, which is indicated by the linearity of $I-V$ characteristics in the Abeles $(\ln (\mathrm{I} / E)-1 / E)$ coordinates (see the insertion in Fig. 3) [5].

Fig. 4 shows the typical $C-V$ characteristic of the investigated structure, where the sign of voltage on the abscissa axis corresponds to the sign of bias on the $\mathrm{M} / \mathrm{nc}-\mathrm{Si}$ contact. The characteristic regions of highfrequency $C-V$ of the MOS structure are visible in the capacity-voltage $\left(C / C_{0}-U\right)$ dependence. At a negative bias, the horizontal capacity line corresponding to the accumulation of holes in the space charge region (SCR) of $p$-Si semiconductor at the interface with nc-Si is observed. Total capacity of the structure under accumulation is determined by the capacity of the nc-Si layer, $C_{\mathrm{nc}-\mathrm{Si}}$, that is less in comparison with the differential capacity of SCR of $p-\mathrm{Si}, C_{\mathrm{D}}$. A growth of the bias in the positive direction corresponds to the subsequent realization of depletion and inversion conditions with the observed sharp drop of capacity.

Flat band voltages $V_{\mathrm{FB}}$ for structures with the undoped nc-Si film have small negative values (about $1 \mathrm{~V})$. For structures with nc-Si films doped with gold, the $V_{\mathrm{FB}}$ values are about $0 \mathrm{~V}$ with lower dispersion.

Using the values of flat band voltages, the effective built-in charge in nc-Si films was calculated by the expression

$Q_{o}=\frac{C_{m}}{S}\left(\varphi_{m s}-V_{\mathrm{FB}}\right)$,

where $\varphi_{m s}$ is the contact difference of metal (Al) and semiconductor $(\mathrm{Si})$ potentials; $S$ is the area of the metal gate electrode, and $C_{m}$ is the maximal capacity of the structure.

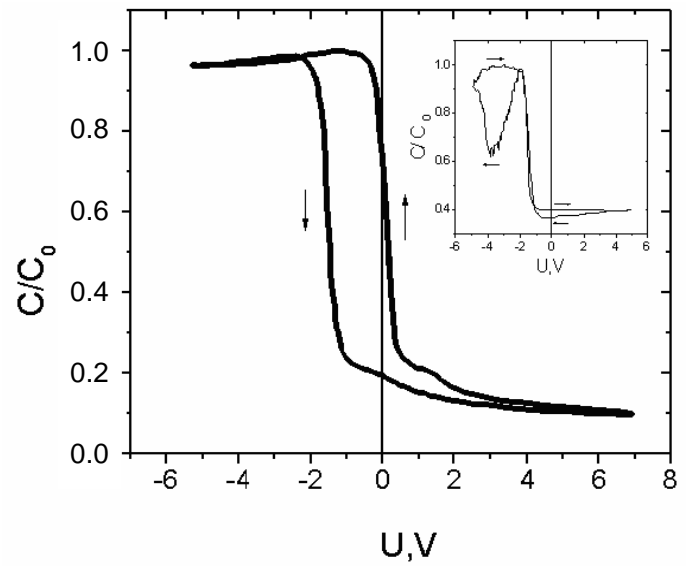

Fig. 4. $C-V$ characteristics of the MIS structure with nanocomposite $\mathrm{Si} \mathrm{NC}-\mathrm{SiO} 2$ film. In insert: $C-V$ characteristic with the capacity decreasing under accumulation.

Calculations showed that the effective built-in charge is positive in the structures with undoped nc-Si films, whereas it is negative in the structures with films doped by gold (Fig. 5a). A positive charge is, usually, connected with $E^{\prime}$ centers (dangling silicon bonds), which arise at the oxygen deficit in a $\mathrm{SiO}_{2}$ film, and with the oxygen vacancies which are deep hole traps [6]. As to a negative charge, the non-bridge oxygen, in particular, can be a cause $\left(\mathrm{O}_{3} \equiv \mathrm{Si}-\mathrm{O}^{-}\right.$centers $)$[7] .

Composition of the undoped nc-Si films is characterized by the $\mathrm{SiO}_{\mathrm{x}}$ suboxides with the presence of large concentrations of oxygen vacancies [8]. As was shown in work [3], the doping of $n c$-Si films with gold results in the effective saturation of dangling silicon bonds on the $\mathrm{Si} \mathrm{NC}$ surface and in the $\mathrm{SiO}_{x}$ matrix. The gold atoms act as catalysts for the $\mathrm{SiO}_{x}$ to the $\mathrm{SiO}_{2}$ oxidization process. As a result, the concentration of oxygen vacancies is reduced, and the concentration of $\mathrm{Si}-\mathrm{O}$ bonds is increased. These processes determine the observed the signs of built-in charges.

The hysteresis loop was observed at recording $C-V$ characteristics in the forward and reverse directions. The negative sign of flat band voltage shift $\left(\Delta V_{\mathrm{FB}}\right)$ points out to the accumulation of effective positive charge in the nc-Si film (see Fig. 4). The values of this charge calculated according to the relation

$\Delta Q=C_{m} \cdot \Delta V_{\mathrm{FB}}$

as functions of the thickness of nc-Si films and the electric field strength are shown in Figs. $5 \mathrm{~b}$ and 6, respectively. As can be seen from Fig. $5 \mathrm{~b}$, the value $\Delta Q$ is higher for the structures with thinner films. Higher accumulation charge in thin films can be caused by less size of $\mathrm{NC}$ and their higher concentration (see the insert in Fig. 1). Growth of $\Delta Q$ in the films doped with gold can be explained by a large content of negatively 

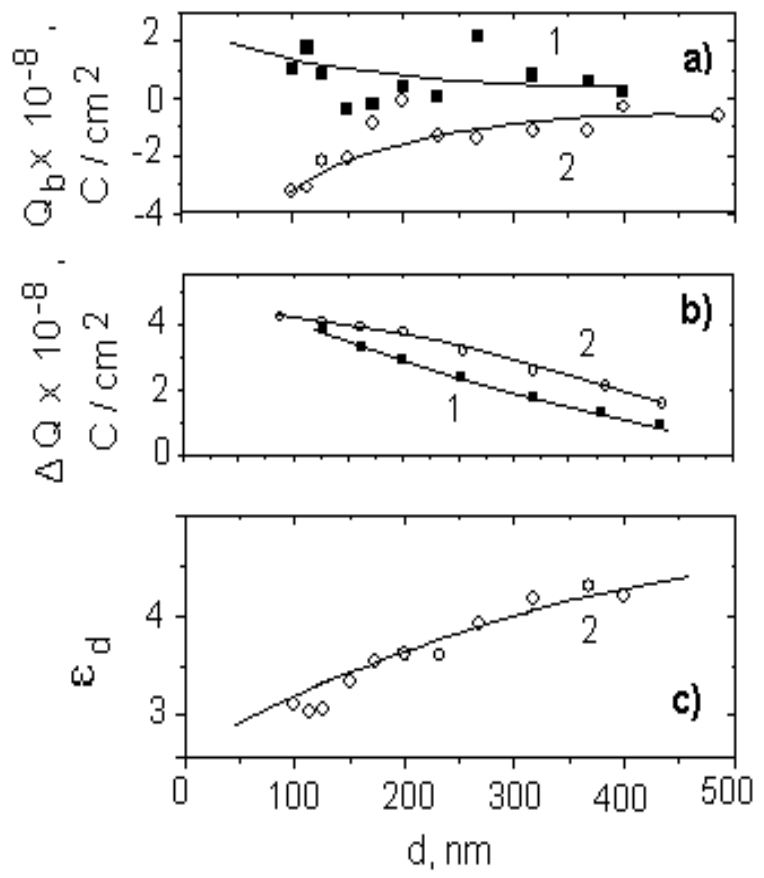

Fig. 5. Built-in charge (a), accumulated charge (b), and relative dielectric permittivity (c) vs nanocomposite film thickness: $1-$ undoped film; 2 - gold-doped film.

charged defects in them caused by non-bridge oxygen. As it was expected, the increase of the accumulated positive charge with the electric field is observed (Fig. 6).

Additional measurements of $C-V$ characteristics with variation of the amplitude of a negative (positive) bias at a fixed value of the opposite-sign bias were performed. With increase in the negative bias, the considerable accumulation of positive charge took place, while only a small negative charge was accumulated with increase in the positive bias. Thus, the positive charge caused by the hole injection from $p$-Si SCR is accumulated in the nc-Si film. The barrier height for holes at the nc-Si / $p$-Si interface is high enough (about $4.6 \mathrm{eV}$ ) in comparison with that of the barrier for electrons. The direct tunneling of holes is complicated in this case, but the mechanism of trap-assisted resonant tunneling through traps in nc-Si is possible $[9,10]$. The current under such a mechanism by orders exceeds the current of direct tunneling. The high-energy barrier for holes promotes increasing the storage time of the accumulated charge.

Reduction of capacity, sometimes with sharp oscillations, under charge accumulation in $p$-Si SCR is a characteristic feature of the measured $C-V$ characteristics (see the insert in Fig. 4). The Si NC charging at the increase of a negative bias causes the given effect. It is obvious that the electric field strength is sufficient for the tunneling of holes from $p$-Si in Si NC. At the capture of charges on $\mathrm{Si} \mathrm{NC}$, the capacity $C_{\mathrm{nc}-\mathrm{Si}}$ is decreased,

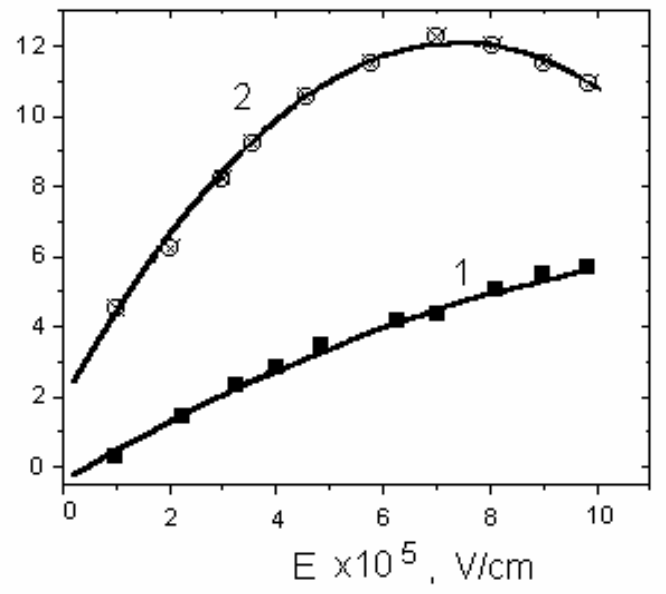

Fig. 6. Accumulated charge $v s$ electric field strength $(d=$ $100 \mathrm{~nm}$ ): 1 - undoped film; 2 - gold-doped film.

because the number of the electronic states reactive on a small variable signal at the $C-V$ measurements is diminished. At the reverse direction of the bias, a capacity is higher than that at the direct direction, which is caused by reduction of the negative bias and, as a result, the release of holes from Si NC. The features of capacity relaxation are determined by statistical fluctuations of the potential on heterointerfaces, dispersion of the characteristic time of defects, and the Si NC recharging in nc-Si films.

Because the maximal capacity of the structure under accumulation is determined by the capacity of the nc-Si film, the relative dielectric permittivity of films $\left(\varepsilon_{d}\right)$ can be calculated from the relation $C_{m}=\varepsilon_{d} \varepsilon_{0} S / d$. In the case of undoped films, the thickness-related dispersion of $\varepsilon_{d}$ is high enough. For the films doped with gold, the values of $\varepsilon_{d}$ increase with increase in the thickness and lie in the range 3 to 5 (Fig. 5c). The large dispersion of $\varepsilon_{\mathrm{d}}$ in the undoped films is explained by considerable irregularities of the structure, and by a higher concentration of pores. In the strongly oxidized gold-doped films, the $\mathrm{SiO}_{2}$ phase fills many pores, and the concentration of large $\mathrm{Si} \mathrm{NC}$ is also increased with thickness. All of these determine the increase of $\varepsilon_{\mathrm{d}}$ with thickness.

Thus, the researches of $C-V$ characteristics of the $\mathrm{Al} / \mathrm{SiO}_{2}$ film with $\mathrm{Si} \mathrm{NC} / p$-Si/Al structures, obtained by pulsed laser deposition of the film on silicon, have shown that the structures possess the hysteresis of $C-V$ characteristics at small voltages and reveal the capture of the accumulated positive charge. It is established that the accumulation of charge is determined by the injection of holes from $p$-Si. Study of the influence of the formation conditions and the parameters of nc-Si films on their charge characteristics allows us to determine that the positive charge is built in the undoped nc-Si films, and the films doped with gold contain the negative charge. Higher accumulation of charge is observed in thinner

\section{(C) 2007, V. Lashkaryov Institute of Semiconductor Physics, National Academy of Sciences of Ukraine}


films and in the films doped with gold. The results are explained by the dependence of the capture efficiency of holes on Si NC, their sizes and density, the influence of gold on the Si NC density, and the defect composition of films. On the other hand, they testify the potential possibility for creation of non-volatile memory devices based on structures containing a dielectric with $\mathrm{Si}$ nanocrystals formed by the pulsed laser deposition method.

\section{References}

1. E.B. Kaganovich, E.G. Manoilov, S.V. Svechnikov, Capacity properties of the structures with lowdimensional silicon // Optoelectronics and semiconductor techniques, No 4, p. 61-80 (2006) (in Russian).

2. E.B. Kaganovich, S.V. Svechnikov, E.G. Manoilov, S.P. Dikiy, L.L. Fedorenko, Photoluminescence of $\mathrm{Si}$ nanocomposite films // Proc. VI Intern. Symp. "Thin films in electronics". MoscowKyiv-Kherson, Naukova dumka, Kyiv, p. 117-119 (1995) (in Russian).

3. E.B. Kaganovich, E.G. Manoilov, I.P. Bazylyuk, S.V. Svechnikov, Photoluminescence spectra of silicon nanocrystals // Physics and Techniques of Semiconductors 37(3), p. 353-357 (2003) (in Russian).
4. P.M. Lytvin, O.S. Lytvin, I.P. Prokopenko, E.B. Kaganovich, I.M. Kizyak, E.G. Manoilov, Structure of photoluminescence films of nanocrystal silicon obtained by pulse laser deposition method // Proc. "Nanosystems, nanomaterials, nanotechnology” 1 (2), p. 601-610 (2004) (in Ukrainian).

5. B. Abeles, P. Sheng, M.D. Coutts, Y Azic, Structural and electrical properties of granular metal films // Adv. Phys. 24 (3) p. 407-461 (1975).

6. D.L. Griscom, Characterization of three $E^{\prime}$-center variants in X-and $\gamma$-irradiated high purity a- $\mathrm{SiO}_{2} / /$ Nucl. Instrum. Methods Phys. Res. B1, p. 481-488 (1984).

7. R.A.B. Devine, The structure of $\mathrm{SiO}_{2}$, its defects and radiation hardness // IEEE Trans. on Nucl. Sci. 41, p. 125-132 (1994).

8. I.P. Lisovsky, V.G. Litovchenko, D.O. Mazunov, E.B. Kaganovich, I.M. Kizyak, E.G. Manoilov, IR spectroscopy of barrier phase of the photoluminescence nanocomposite films $\mathrm{Si} / \mathrm{SiO}_{\mathrm{x}} / /$ Ukr. Phys. J. 50 (6), p. 582-585 (2005).

9. F. Compabadal, V. Milian, X. Aymerich-Humet, Trap-assisted tunneling in MIS and Schottky structures // Phys. status solidi (a), 79(1), p. 223236 (1983).

10. J. Millan, F. Serra-Metres, X. Aymerich-Humet, Resonant tunnel switching in MIS devices // Solid State Electronics, 25(7), p. 565-569 (1982). 